\title{
The influence of open and closed high tibial osteotomy on dynamic patellar tracking: a biomechanical study
}

\author{
Robert Gaasbeek · Roy Welsing • Marco Barink • \\ Nico Verdonschot · Albert van Kampen
}

Received: 27 September 2006/ Accepted: 30 January 2007/Published online: 5 May 2007

(C) Springer-Verlag 2007

\begin{abstract}
High tibial osteotomy (HTO) can cause alterations in patellar height, depending on the surgical technique, the amount of correction and the postoperative management. Alterations in patella location after HTO may lead to postoperative complications. However, information on changes in dynamic patellar kinematics following HTO is very limited. We conducted a biomechanical study, to analyze the effect of open (OWO) and closed wedge osteotomy (CWO) on patellar tracking. Using an inventive experimental setup, we studied the 3D dynamic patellar tracking in ten cadaver knees before and after valgus HTO. In each specimen, corrections of $7^{\circ}$ and $15^{\circ}$ of valgus according to, both, the OWO and CWO technique, were performed. Patellar height significantly increased with CWO and decreased with OWO. Both, OWO and CWO led to significant changes in the patellar tracking parameters tilt and rotation. We also found significant differences between OWO and CWO. Valgus high tibial osteotomy increased the medial patellar tilt and
\end{abstract}

R. Gaasbeek $(\bowtie)$

Department of Orthopedics and Traumatology,

Meander Medical Center, P.O. Box 1502,

3800 BM Amersfoort, The Netherlands

e-mail: rda.gaasbeek@meandermc.nl

A. van Kampen

Department of Orthopaedics,

Radboud University Nijmegen Medical Center,

Th. Craanenlaan 7, 6525 GH, P.O. Box 9101,

6500 HB Nijmegen, The Netherlands

R. Welsing - M. Barink · N. Verdonschot

Orthopaedic Research Laboratory,

Radboud University Nijmegen Medical Center,

Th. Craanenlaan 7, 6525 GH, P.O. Box 9101,

6500 HB Nijmegen, The Netherlands reduced the medial patellar rotation. These effects were more profound after OWO. No significant differences were found for the effect on medial-lateral patellar translation. These observations can be taken into consideration in the decision whether to perform an OWO or a CWO in a patient with medial compartment osteoarthritis of the knee.

Keywords High tibial osteotomy - Patellar tracking · Osteoarthtritis $\cdot$ Knee $\cdot$ Kinematics

\section{Introduction}

Young active patients suffering from medial compartment osteoarthritis of the knee with a varus alignment can be treated with a high tibial osteotomy (HTO). Depending on the surgical technique and postoperative management, alterations in patellar height can be induced $[2,3,6,11,14,16,21]$. Open wedge osteotomy (OWO) proximal to the tibial tuberosity, which is gaining popularity in recent years, has been reported to cause patella baja $[6,12,21]$. Closed wedge osteotomy (CWO) has been shown to lead to both patella baja and alta $[6,12]$. These alterations in patellar height have been attributed to the proximalisation (CWO) or distalisation (OWO) of the tibial tuberosity following HTO [21]. Furthermore, scarring, adhesions and contracture of the patella ligament are mentioned to cause lowering of the patella in CWO [14]. Besides these changes in patellar height, other components of patellar tracking may be affected by HTO. This may cause anterior knee pain, patella locking, crepitus and limitation of knee motion. Eventually, the altered patellofemoral congruency and contact stress may lead 
to patellofemoral osteoarthritis. Furthermore, there is evidence that patellar height may affect the outcome of total knee arthroplasty $[5,20]$. Conversely, pre-existing patellofemoral symptoms may diminish following HTO [7].

Former studies on the effect of HTO on the patellofemoral joint focus on the standard plain radiographic measurements of patellar height at one position of knee flexion [2,12]. Furthermore, previous studies in this field restrict the dynamic patellar tracking to a range of static measurements of the patella position in a few fixed positions of knee flexion $[8,19]$. As far as the authors are aware of, no study has been reported that describes the true dynamic patellar tracking following HTO. The present study was conducted to analyse the changes in dynamic patellar tracking after both, OWO and CWO.

\section{Materials and methods}

\section{Specimens}

Ten fresh frozen cadaver knees were obtained. Degenerative changes and dysplasias were evaluated and excluded based on standard AP, lateral and patella radiographs. The specimens were prepared for use in a knee joint motion and loading apparatus [17]. Therefore, tibia and femur were transsectioned at about $20 \mathrm{~cm}$ from the knee joint centre. The ends of the bones were potted in autopolymer, which guaranteed identical positioning during the different surgeries and measurements. All soft tissues were preserved. The quadriceps muscle was separated in three parts: rectus femoris, vastus medialis, and vastus lateralis/intermedius. Three mounting plates were rigidly fixed to the femur, tibia and patella, respectively, which enabled the registration of the bony segments visible on the CT scans to the global coordinate system. Hereafter, CT scans of all knees were obtained.

\section{Experimental set up}

A specially designed metal frame with hinges on the medial and lateral side was put on the tibia under fluoroscopic view. This frame was designed such that the position of the hinges coincided with the planned rotation point (the apex) of the osteotomy. The frame allowed a free moving distal part of the tibia. In this way, different valgus angles within the same specimen could be simulated. The tibia positions could be set at a predetermined angle.
After this, the specimen was positioned in the loading apparatus. An electromagnetic motion tracking system (3SPACE Fastrak, Polhemus, Colchester, VT, USA) was used to measure the patello-femoral and tibio-femoral kinematics of the knee. Three sensors were fixed rigidly to the mounting plates on femur, tibia and patella. The kinematics were recorded using continuous data acquisition. The three separated parts of the quadriceps muscle were loaded with $27 \mathrm{~N}$ each and an additional $50 \mathrm{~N}$ axial compressive load was applied to the knee, according to an earlier published protocol [17, 18]. For the first reference measurement, three flexion-extension movements were performed manually. Next, a standard anterolateral approach was used. Two centimetres below and parallel to the joint line a Kirschner wire was inserted to aim the osteotomy. First, the proximal cut was performed. Using an aiming device the distal cut was made and a $15^{\circ}$ bony wedge was removed proximal to the tibial tuberosity. The fibula was cut, according to the closed wedge HTO technique of Coventry [4].

The knee was put back into the loading apparatus and the measurements were repeated in three different positions of the distal tibia: neutral (Fig. 1a), $7^{\circ}$ and $15^{\circ}$ valgus (lateral closed wedge) position (Fig. 1b).

After this, a medial approach was performed, shifting the pes anserinus and the superficial medial collateral ligament dorsally. A Kirschner wire was inserted parallel to the joint line. Using a guide instrument a second wire was inserted just proximal of the tuberosity in lateral cranial direction in order to obtain an equal osteotomy level in all knees. Along this wire the osteotomy was performed.

Hereafter, flexion-extension cycles were recorded in neutral, $7^{\circ}$ and $15^{\circ}$ valgus (medial open wedge) position (Fig. 1c). All correction angles were checked with an electronic inclinometer (Cybex, EDI 320). The surgery was performed under fluoroscopic view.

\section{Data analysis}

After the recordings were made, the Fastrak motion data were analysed and combined with the CT-scanning data. The 3D motion data, which were measured in the global coordinate system, were transformed to anatomic coordinates. The anatomic coordinate systems were based on bony landmarks, which were retrieved from the CT scan contours [1]. In this way, a 3D dynamic model of each knee was constructed with a local anatomic coordinate system. As the patellar kinematics are also largely dependent on the tibial movements the tibial kinematics were also measured. The calculated patellar tracking parameters were: 

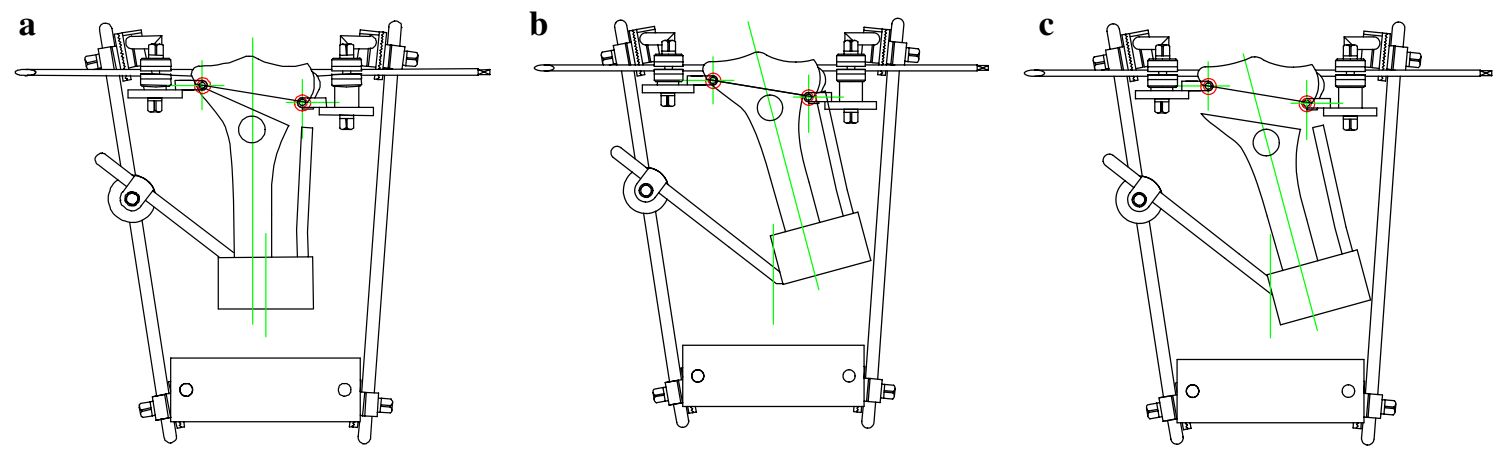

Fig. 1 Schematic presentation of the tibia positioned in the specially designed frame which enabled testing of different valgus angles within the same specimen. a Neutral, b valgus closed wedge $\left(7^{\circ}\right.$ and $\left.15^{\circ}\right)$ and $\mathbf{c}$ valgus open wedge $\left(7^{\circ}\right.$ and $\left.15^{\circ}\right)$

patellar height, tilt, rotation, and lateral-medial translation (For definition of parameters see Fig. 2). To quantify the impact of the HTO for each of the parameters, the differences of the patellar kinematics after HTO minus the reference patellar kinematics of the neutral measurements were calculated.

\section{Statistics}

The three reference measurements in neutral position were compared using a Two-way ANOVA. The differences between the four groups, e.g. $7^{\circ}$ and $15^{\circ}$ lateral closed wedge, and $7^{\circ}$ and $15^{\circ}$ medial open wedge, were analyzed by using a Two-way ANOVA at every subsequent flexion step of $5^{\circ}$ up to maximal knee flexion angle of $100^{\circ}$. To assess whether each group was statistically different from the non-operated situation, we calculated whether the difference of the two measurements (intact minus operated) was different from zero by calculating if the value zero was within the outcome of the $95 \%$ confidence interval. Significance was set at $P<0.05$.

\section{Results}

In general, the movement patterns of the patella in the non-operated knees were similar to what clinically can be expected and is described by van Kampen and Huiskes [18]. Typical examples of the effects of HTO on patellar tilt and rotation in one of the specimen are shown in Fig. 3a and b, respectively. The group results are graphically displayed in Fig. 4a-d. They represent the change of patellar kinematics caused by the HTO, not the absolute values.

Patellar height

As expected, larger tibial correction angles induced more patellar height changes (Fig. 4a). The OWO resulted in significant lowering of the patella. Larger corrections in the OWO group caused significant more patella lowering, whereas more correction in the $\mathrm{CWO}$ group resulted in more increase in patellar height. The change of patellar height was not related to the knee flexion. Hence, during knee flexion the position of the patella in relation to the tibia was rather constant, which resulted in horizontal curves. The mean decrease of patellar height following $7^{\circ}$ OWO was $1.5 \mathrm{~mm}$ (range $0.7 ; 1.7$ ) which significantly increased to $4.2 \mathrm{~mm}$ (range $2.7 ; 4.6$ ) at $15^{\circ} \mathrm{OWO}$. The patellar height after $7^{\circ} \mathrm{CWO}$ increased on average with $2.2 \mathrm{~mm}$ (range 1.1;2.7). At $15^{\circ} \mathrm{CWO}$ the mean increase was $3.0 \mathrm{~mm}$ (range 2.1;3.6). Figure 5 shows a schematic presentation of the patellar height changes after open an closed wedge osteotomy.
Fig. 2 Definition of patellar movements
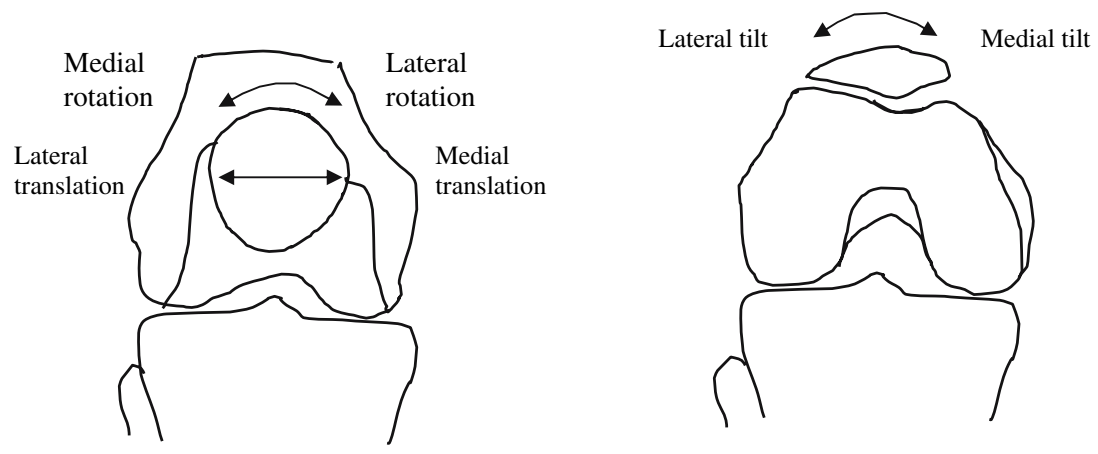

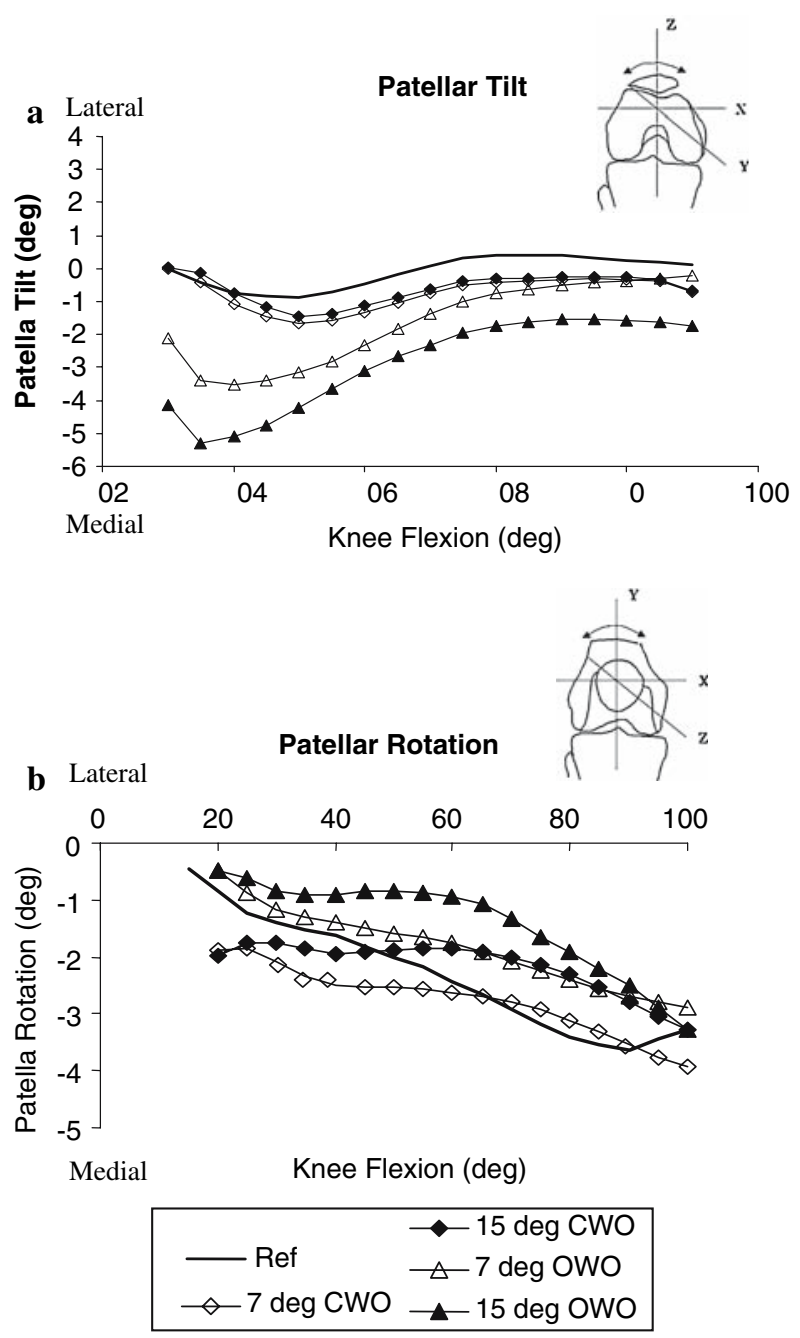

Fig. 3 Typical examples of the effects of HTO on the patellar tilt (a) and rotation (b) of one specimen. Ref: Reference curve of neutral measurement

\section{Patellar tilt}

A typical example of the effects of HTO on the patellar tilt is shown in Fig. 3a. On average, OWO and CWO caused the patella to tilt more medially (Fig. 4b). A trend was seen of more medial tilt with OWO than with CWO. Along the flexion-extension movement we found no significant difference between CWO and OWO at $7^{\circ}$ correction, but during the first half of flexion significant differences were found between $\mathrm{CWO}$ and $\mathrm{OWO}$ with a $15^{\circ}$ correction. OWO caused the patella to tilt more medially in extension and showed a decrease in additional medial tilt while the knee was brought in flexion. With increasing flexion the difference in medial tilt between the two HTO groups decreased. All groups showed to be significant different from zero. The mean increase of medial tilt following $7^{\circ} \mathrm{OWO}$ was $1.7^{\circ}$ (range $0.3 ; 4.8$ ), which increased to $2.9^{\circ}$ (range $1.8 ; 6.2$ ) at $15^{\circ}$ OWO. The mean medial patellar tilt after $7^{\circ} \mathrm{CWO}$ increased with $0.7^{\circ}$ (range $0.0 ; 0.9$ ). At $15^{\circ} \mathrm{CWO}$ the mean increase was $0.5^{\circ}$ (range $-0.3 ; 0.7$ ).

Patellar rotation

A typical example of the effects of HTO on the patellar rotation is shown in Fig. 3b. Both, OWO and CWO reduced the normal medial rotation of the patella along the flexion movement of the knee. In general, OWO showed significant more reduction of medial rotation than CWO (Fig. 4c). No statistical difference between $\mathrm{CWO}$ and $\mathrm{OWO}$ with $7^{\circ}$ correction was found around the area in the curves where the two lines cross. At $7^{\circ} \mathrm{OWO}$ correction, the reduction of the medial rotation declined with increased flexion of the knee. In full flexion this group showed a small increase of medial rotation of the patella. Increasing the correction angle from $7^{\circ}$ to $15^{\circ}$ resulted in a significant reduction of medial rotation and in some specimen lateral rotation was induced. No significant difference was found between CWO 7 and $15^{\circ}$. During the first half of flexion movement the effect of CWO on the patellar rotation was not significantly different from zero, and this was also seen for the $7^{\circ}$ of correction with OWO near full extension. The mean reduction of medial rotation following $7^{\circ}$ OWO was $1.6^{\circ}$ (range $-0.6 ; 2.8$ ), which increased to $5.2^{\circ}$ (range $4.7 ; 5.5$ ) at $15^{\circ}$ OWO. The mean medial rotation after $7^{\circ} \mathrm{CWO}$ decreased with $1.2^{\circ}$ (range $-0.1 ; 1.9$ ). At $15^{\circ} \mathrm{CWO}$ the mean reduction was $1.1^{\circ}$ (range $0.0-1.9$ ).

Patellar medial-lateral translation

We found a poor reproducibility of the differences in medial-lateral patellar translation leading to high standard deviations. No significant differences were found between groups in medial or lateral translation of the patella $(P>0.05)$. Also, all calculated effects were not different from the reference patellar-tracking pattern (Fig. 4d).

\section{Discussion}

Previous observations of patellar height after HTO concern either the closed wedge or the open wedge technique. To our knowledge, only three studies analyzed the effects on patellar height in OWO versus CWO. Tigani et al. [16] used Caton's Index (CI) and showed lowering of the patella more often with OWO than with $\mathrm{CWO}$ and a high degree of patella 


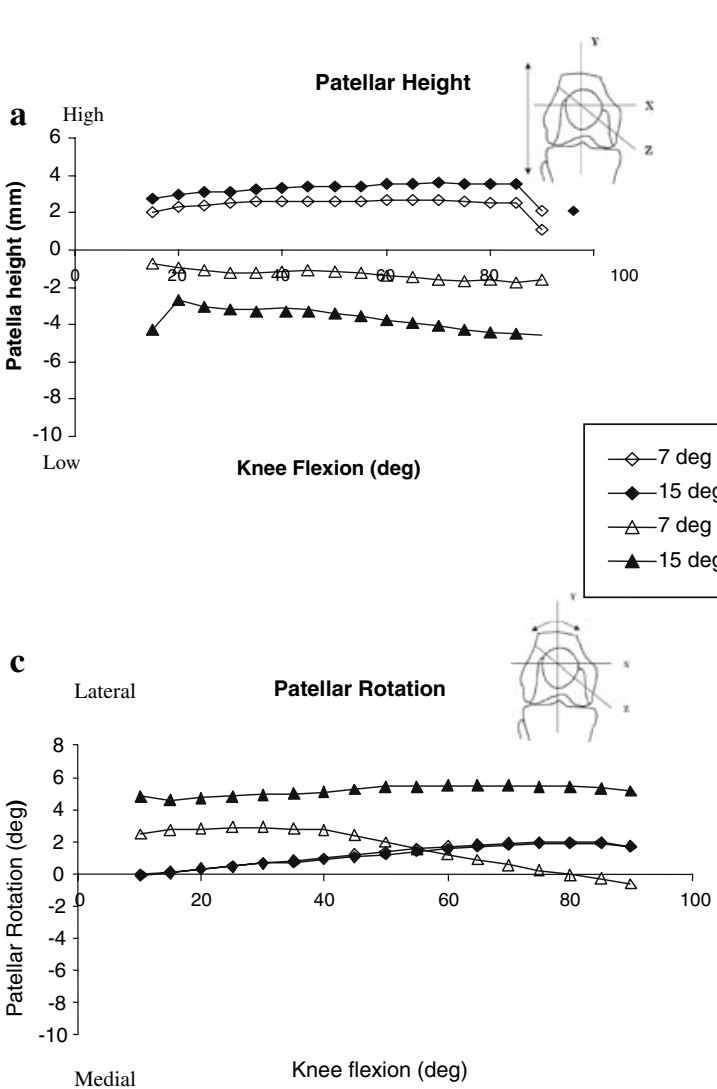

Fig. 4 Averaged group patellar kinematics: patellar height (a), patellar tilt (b), patellar rotation (c) and patellar medial-lateral translation (d). The data represent the change of patellar

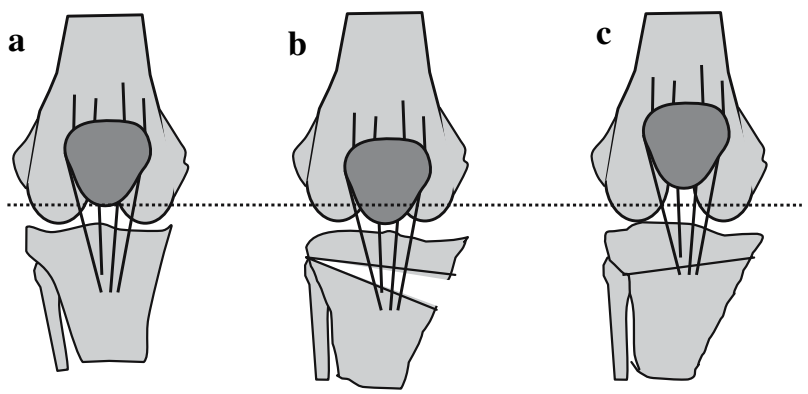

Fig. 5 Schematic presentation of the changes in patellar height after high tibial osteotomy. a Preoperative, b lowering of the patella after open wedge osteotomy and $\mathbf{c}$ increased patellar height after closed wedge osteotomy

proximalisation in the latter. Brouwer et al. [2] found more patellar lowering after an open wedge HTO. They applied both the Insall Salvati (ISI) and the Blackburne-Peel Index (BPI). Hoell et al. [9] measured patellar height change with the ISI and found no difference between OWO and CWO. However, this does not exclude a true change of patellar height in relation to the joint line, as the ISI represents the length of the patellar tendon. Furthermore, BPI and CI
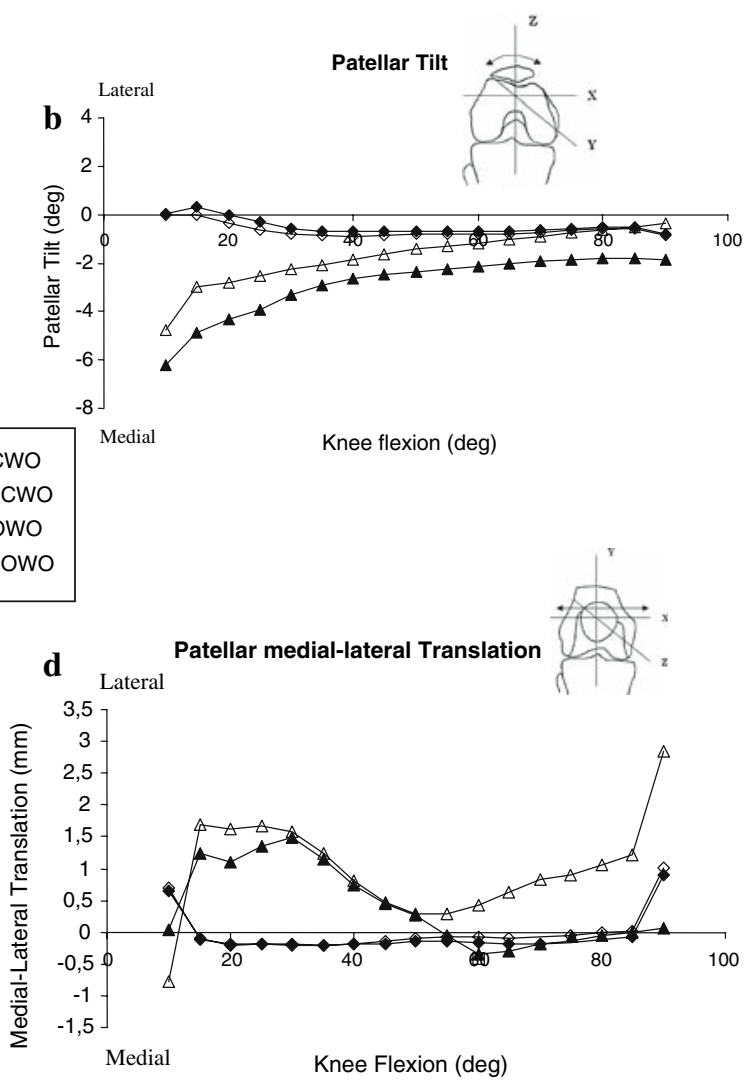

tracking parameters caused by the different HTO techniques, not the absolute values. Because of clarity standard deviations are not shown

do not accurately measure the alteration of patellar height after valgus HTO, because they are dependent of the tibial inclination and the antero-posterior translation of the proximal tibia [12]. The present study has the advantage of continuous 3D monitoring of the patella movements, without the limitations of the measurements of patellar height on plain roentgen films. We found a significant increase in patellar height after CWO and a significant lowering of the patella after OWO. These height changes were dependent on the amount of correction and more profound after OWO as compared to CWO (Fig. 4a). Concerning the biomechanics of the patella movement in relation to its anatomical position contradictory information in literature is found. Investigations of the mechanics of patella baja have found that a low-riding patella does not necessarily lead to an increase in patellofemoral contact forces [13, 15]. However, others report an increase in patellofemoral joint reaction force as a result of adhesions of the patella ligament [21].

During surgery we observed that the proximal translation after CWO was limited by the medial and lateral retinaculum to such extend that the patellar 
tendon relaxed and curled up. This phenomenon might well be one of the factors that lead to shortening and adhesions of the patellar tendon that is reported after CWO [14].

Weidenhielm et al. reported on seven patients before and after CWO using kinematic analysis with roentgen stereophotogrammetric analysis (RSA). They found no significant change in patellar rotation and translation during knee motion, with inconsistent changes in patella position [19]. However, they were not informed about quadriceps tension nor did they correct for relaxation or contraction of the quadriceps muscles during the RSA measurements.

This might have been a large confounder in their study. During flexion of the knee, until the patella is well seated, its stability rests solely on muscle tension. Hungerford et al. [10] stated that the congruence of the patellofemoral joint and the muscles forces provide considerable stability irrespective of restraining ligaments. Because of this we applied a constant load to the quadriceps muscle during our experiments (See Material and methods). Hill et al. [8] used magnetic resonance imaging to measure patellofemoral kinematics through a range of loaded flexion before and after CWO in four patients. They found a decrease in patella flexion and internal spin ('medial rotation' in this study), and an increase in medial patellar tilt and proximal translation. Although this study represents a "semi-dynamic" setting, our results corroborate with their findings. We found that valgus osteotomy increased the medial tilt and reduced the medial patellar rotation. These effects were more profound after OWO. The increased medial patellar tilt can be explained by the increased lateral pull on the patella. The tuberosity is located more laterally after valgus high tibial osteotomy. The lateral patellar facet is pressed against the lateral wall of the trochlea and the patella is forced up the lateral groove leading to a medial tilt. As the quadriceps tendon remains to have the same loading direction, the lateralization of the tuberosity induces lateral rotation (reduction of medial rotation). Theoretically, the effect on the patellar medial-lateral translation is larger near full extension of the knee. During flexion the patella enters the trochlear groove, which will enhance medial-lateral stability. Furthermore, there is a relatively high variation in patellar position in the lower range of flexion. These phenomena may explain why no significant differences in patellar translation could be detected. Furthermore, during our experiments we observed that OWO, led to more tension on the patella ligament and the retinaculi in contrast to the closed wedge osteotomies, in which we observed a relative relaxation of the patellar tendon. This may explain why more effect of the HTO was observed in the OWO group. It seemed that the distalisation and increased tension following OWO, enhances the forces at the patellofemoral joint, leading to more obvious effects on the patellar tracking. If these effects are unwanted, a different operation technique has been described recently, to prevent the distalisation of the tibial tuberosity with OWO by performing a distal tuberosity osteotomy [6].

To our knowledge, the current paper is the first to describe the continuous 3D patellar tracking changes following HTO, according to both the CWO as the OWO technique. This study clearly shows the evident influence of HTO on patellar position and the patellofemoral movements. The effects of OWO proof to be more profound than those following CWO, especially at larger valgus corrections of the tibia. These effects of HTO on patellar height and patellar tracking may be of minimal clinical relevance but in extensive open wedge corrections the effects may be quite large and lead to adverse events with respect to the patellofemoral joint (e.g. pain, maltracking or even patellar (sub)luxation). On the other hand, patellofemoral complaints may subside after high tibial osteotomy.

In conclusion, the decision whether a closed or an open wedge technique is performed, for the treatment of patients suffering from medial osteoarthritis of the knee, the presence of patellofemoral complaints or pre-existent patella baja should be taken into consideration. Especially in large open wedge corrections significant changes in patellar height and tracking can be induced. In the future, the effect of the kinematic changes in the patellofemoral joint after high tibial osteotomy in terms of clinical presentation and radiographic changes after long term follow-up have to be determined.

\section{References}

1. Barink M, Meijerink H, Verdonschot N, van Kampen A, de Waal MM (2006) Asymmetrical total knee arthroplasty does not improve patella tracking: a study without patella resurfacing. Knee Surg Sports Traumatol Arthrosc [Epub ahead of print]

2. Brouwer RW, Bierma-Zeinstra SM, van Koeveringe AJ, Verhaar JA (2005) Patellar height and the inclination of the tibial plateau after high tibial osteotomy. The open versus the closed-wedge technique. J Bone Joint Surg Br 87:12271232

3. Closkey RF, Windsor RE (2001) Alterations in the patella after a high tibial or distal femoral osteotomy. Clin Orthop 389:51-56

4. Coventry MB (1965) Osteotomy of the upper portion of the tibia for degenerative arthritis of the knee. A preliminary report. J Bone Joint Surg Am 47:984-990 
5. Figgie HE III, Goldberg VM, Heiple KG, Moller HS III, Gordon NH (1986) The influence of tibial-patellofemoral location on function of the knee in patients with the posterior stabilized condylar knee prosthesis. J Bone Joint Surg Am 68:1035-1040

6. Gaasbeek RD, Sonneveld H, van Heerwaarden RJ, Jacobs WC, Wymenga AB (2004) Distal tuberosity osteotomy in open wedge high tibial osteotomy can prevent patella infera: a new technique. Knee 11:457-461

7. Goutallier D, Delepine G, Debeyre J (1979) L'articulation fémoro-patellaire dans le genu-arthrosique. Rev Chir Orthop Reparatrice Appar Mot 65:25-31

8. Hill NA, Fellows RA, MacIntyre NJ, Harrison MM, Ellis RE, Wilson DR (2005) The effect of high tibial osteotomy on three-dimensinal tibiofemoral and patellofemoral kinematics: an in vivo study using magnetic resonance imaging. In: Proceedings of 51st ORS conference 0481

9. Hoell S, Suttmoeller J, Stoll V, Fuchs S, Gosheger G (2005) The high tibial osteotomy, open versus closed wedge, a comparison of methods in 108 patients. Arch Orthop Trauma Surg 125(9):638-643

10. Hungerford DS, Barry M (1979) Biomechanics of the patellofemoral joint. Clin Orthop Relat Res 144:9-15

11. Kaper BP, Bourne RB, Rorabeck CH, Macdonald SJ (2001) Patellar infera after high tibial osteotomy. J Arthroplasty 16:168-173

12. Kesmezacar H, Erginer R, Ogut T, Seyahi A, Babacan M, Tenekecioglu Y (2005) Evaluation of patellar height and measurement methods after valgus high tibial osteotomy. Knee Surg Sports Traumatol Arthrosc 13(7):539-544
13. Meyer SA, Brown TD, Pedersen DR, Albright JP (1997) Retropatellar contact stress in simulated patella infera. Am J Knee Surg 10:129-138

14. Scuderi GR, Windsor RE, Insall JN (1989) Observations on patellar height after proximal tibial osteotomy. J Bone Joint Surg Am 71:245-248

15. Singerman R, Davy DT, Goldberg VM (1994) Effects of patella alta and patella infera on patellofemoral contact forces. J Biomech 27:1059-1065

16. Tigani D, Ferrari D, Trentani P, Barbanti-Brodano G, Trentani F (2001) Patellar height after high tibial osteotomy. Int Orthop 24:331-334

17. Van Heerwaarden HJ (1998) Effects of pretension in reconstructions of the anterior cruciate ligament; clinical, biomechanical and computer model analysis. Thesis/Dissertation, University of Nijmegen

18. van Kampen A, Huiskes R (1990) The three-dimensional tracking pattern of the human patella. J Orthop Res 8:372382

19. Weidenhielm L, Wykman A, Lundberg A, Brostrom LA (1993) Knee motion after tibial osteotomy for arthrosis. Kinematic analysis of 7 patients. Acta Orthop Scand 64:317319

20. Windsor RE, Insall JN, Vince KG (1988) Technical considerations of total knee arthroplasty after proximal tibial osteotomy. J Bone Joint Surg Am 70:547-555

21. Wright JM, Heavrin B, Begg M, Sakyrd G, Sterett W (2001) Observations on patellar height following opening wedge proximal tibial osteotomy. Am J Knee Surg 14:163-173 